\title{
Lectures on the Cohomology of Finite Groups
}

\author{
Alejandro Adem* \\ ABstract. These are notes based on lectures given at the summer school \\ "Interactions Between Homotopy Theory and Algebra", which was held at the \\ University of Chicago in the summer of 2004.
}

\section{Introduction}

Finite groups can be studied as groups of symmetries in different contexts. For example, they can be considered as groups of permutations or as groups of matrices. In topology we like to think of groups as transformations of interesting topological spaces, which is a natural extension of the classical problem of describing symmetries of geometric shapes. It turns out that in order to undertake a systematic analysis of this, we must make use of the tools of homological algebra and algebraic topology. The context for this is the cohomology of finite groups, a subject which straddles algebra and topology. Groups can be studied homologically through their associated group algebras, and in turn this can be connected to the geometry of certain topological spaces known as classifying spaces. These spaces also play the role of building blocks for stable homotopy theory and they are ubiquitous in algebraic topology.

In these notes we have attempted to lay out a blueprint for the study of finite group cohomology which totally intertwines the algebraic and topological aspects of the subject. Although this may pose some technical difficulties to the readers, the advantages surely outweigh any drawbacks, as it allows them to understand the geometric motivations behind many of the constructions.

The notes reflect the content of the lectures given by the author at the summer school Interactions Between Homotopy Theory and Algebra held at the University of Chicago in August 2004. The first talk was essentially an outline of finite group cohomology from a combined algebra/topology point of view; the second talk was a discussion of connections between group cohomology and representation theory via the concept of minimal resolutions; finally the third talk was a discussion of the role played by group cohomology in the study of transformation groups. This is

2000 Mathematics Subject Classification. 20J06, 57525.

Key words and phrases. cohomology of finite groups.

*Partially supported by NSF and NSERC. The author is very grateful to Bill Dwyer for his generous help in preparing this manuscript. 
reflected in the organization of this paper; note that the final section summarizes recent work by the author and others on constructing free group actions on a product of two spheres. The style is somewhat terse, as the goal was to expose the summer school participants to research topics in the subject, beyond the usual background material. However, there are several excellent books on the cohomology of finite groups (listed in the bibliography), and the hope is that the readers of this very short survey will feel motivated to consult them, as well as the original research papers in the literature.

The author is grateful to the organizers of the summer school for being given the opportunity to give these lectures as well as for their help in preparing this manuscript.

\section{Preliminaries}

We begin by recalling some basic facts about classifying spaces of finite groups and group cohomology; useful references for this material are $[\mathbf{3}],[\mathbf{9}]$ and $[\mathbf{1 3}]$.

Let $G$ denote a finite group and $E G$ a free, contractible $G$-complex (this means that $E G$ is a $C W$-complex on which $G$ acts in a way which freely permutes the cells). The orbit space $B G=E G / G$ is called a classifying space for $G$. The quotient map $E G \rightarrow B G$ is a principal covering space with group $G$, and from this it follows directly that $B G$ is a space of type $K(G, 1)$. In particular, $B G$ is path connected, the fundamental group of $B G$ is isomorphic to $G$, and the higher homotopy groups of $B G$ are trivial. The homotopy type of $B G$ does not depend upon the choice of $E G$.

2.1. REMARK. The term "classifying space" stems from the fact that isomorphism classes of principal $G$ bundles over a CW-complex $B$ correspond bijectively to homotopy classes of maps $B \rightarrow B G$ (see [34]). In other words, such bundles are classified by maps to $B G$. The correspondence sends a map $B \rightarrow B G$ to the pullback over $B$ of the projection $E G \rightarrow B G$.

2.2. Definition. For any $\mathbb{Z}[G]$-module $M$, we define the cohomology groups of $G$ with coefficients in $M$, denoted $H^{i}(G, M)(i \geq 0)$ by the formula

$$
H^{i}(G, M)=H^{i}(B G, M) .
$$

The notation " $H^{i}(B G ; M)$ " above signifies singular (or cellular) cohomology with local coefficients; the local coefficient system is derived from the given action of $G=\pi_{1} B G$ on $M$.

2.3. REMARKS. (1) Let $S_{*}(E G)$ be the integral singular complex of $E G$, so that essentially by definition, $H^{i}(G, M)$ is isomorphic to the $i$ 'th cohomology group of the cochain complex $\operatorname{Hom}_{\mathbb{Z}[G]}\left(S_{*}(E G), M\right)$. The fact that $E G$ is acyclic and that $G$ acts freely on $E G$ implies that the augmentation map $S_{*}(E G) \rightarrow \mathbb{Z}$ gives a free resolution of $\mathbb{Z}$ over $\mathbb{Z}[G]$ (here $\mathbb{Z}$ serves as a trivial $G$-module, i.e., a module on which each element of $G$ acts as the identity). By basic homological algebra, we have

$$
H^{i}(G, M)=\operatorname{Ext}_{\mathbb{Z}[G]}^{i}(\mathbb{Z}, M) \quad(i \geq 0) .
$$

In particular, $H^{0}(G, M)$ is the fixed submodule $M^{G}$, and the higher cohomology groups result from applying to $M$ the higher right derived functors of this fixedpoint construction. 
(2) Using joins, we may construct a model for $E G$ which is functorial in $G$, namely $E G=\operatorname{colim}_{i} G^{* i}$, where $G^{* i}$ is the join $G * G * \cdots * G, i$ times. The points of $E G$ can be thought of as infinite formal sums $\sum_{i>0} t_{i} g_{i}$, where $g_{i} \in G, t_{i} \in[0,1]$, only finitely many $t_{i}$ are nonzero, and $\sum t_{i}=1$. We define a right $G$-action on $E G$ by

$$
\left(\sum_{i} t_{i} g_{i}\right) \cdot g=\sum_{i} t_{i}\left(g_{i} g\right) \text {. }
$$

From this point of view, the space $E G$ is endowed with the smallest topology which makes the coordinate functions

$$
t_{i}: E G \rightarrow[0,1] \quad \text { and } \quad g_{i}: t_{i}^{-1}(0,1] \rightarrow G
$$

continuous. Taking joins increases connectivity, so $E G$ is contractible. It is clear that $G$ acts freely on $E G$, and it is an interesting exercise to construct a $\mathrm{CW}$ structure on $E G$ so that the action of $G$ permutes the cells. If $f: H \rightarrow G$ is a group homomorphism, we get induced maps $E H \rightarrow E G$ and $B H \rightarrow B G$, as well as maps $H^{i}(G, M) \rightarrow H^{i}\left(H,\left.M\right|_{H}\right)$. Here $\left.M\right|_{H}$ is $M$, treated as an $\mathbb{Z}[H]$-module by means of the ring homomorphism $\mathbb{Z}[f]: \mathbb{Z}[H] \rightarrow \mathbb{Z}[G]$.

(3) If $R$ is a ring with a trivial action of $G$, then $H^{*}(G, R)$ has a natural graded multiplicative structure, arising from the cup product.

(4) If $\mathbb{F}_{p}$ is given the trivial $G$-module structure, then $H^{*}\left(G, \mathbb{F}_{p}\right)$ is the ordinary $\bmod p$ cohomology ring of $B G$, and so it has an action of the Steenrod algebra $\mathcal{A}_{p}$.

(5) The low dimensional cohomology groups $H^{i}(G ; M), i=1,2$ arose classically in the study of extensions of $G$ by $M$. Such an extension is a short exact sequence

$$
1 \rightarrow M \rightarrow \tilde{G} \rightarrow G \rightarrow 1
$$

in which the conjugation action of $\tilde{G}$ on $M$ induces the given $G$-module structure on $M$. Isomorphism classes of these extensions correspond bijectively to $H^{2}(G, M)$. If the extension corresponds to the zero cohomology class, then there is a section $G \rightarrow \tilde{G}$, and $H^{1}(G, M)$ acts freely and transitively on the set of $M$-conjugacy classes of such sections.

The group $H^{3}(G ; M)$ arises in a similar but more complicated context in studying extensions of $G$ by a nonabelian group $H$; the relevant $M$ is the center of $H$, and elements of $H^{3}(G, M)$ come up as obstructions to realizing a map from $G$ to the outer automorphism group of $H$ via an extension.

2.4. ExAmples. (1) If $G=\mathbb{Z} / 2$, then $B G$ is equivalent to $\mathbb{R} P^{\infty}$, and $H^{*}\left(B G, \mathbb{F}_{2}\right)$ is a polynomial algebra $\mathbb{F}_{2}[x]$ with $|x|=1$.

(2) If $G=\mathbb{Z} / p$ with $p$ odd, then $B G$ is equivalent to the infinite lens space $L_{p}^{\infty}=$ $\operatorname{colim}_{i} S^{2 i-1} / \mu_{p}$, and $H^{*}\left(B G, \mathbb{F}_{p}\right)$ is the tensor product $\Lambda(x) \otimes \mathbb{F}_{p}[y]$ of an exterior algebra and a polynomial algebra, where $|x|=1$ and $|y|=2$. In this case $y$ is the Bockstein $\beta(x)$.

(3) It is easy to see that $E(G \times H)$ can be taken to be $E G \times E H$, so that $B(G \times H)$ is homotopy equivalent to $B G \times B H$. By the Künneth formula, then, there are isomorphisms

$$
H^{*}\left((\mathbb{Z} / p)^{n}, \mathbb{F}_{p}\right) \cong \begin{cases}\mathbb{F}_{p}\left[x_{1}, \ldots, x_{n}\right] & p=2 \\ \Lambda\left(x_{1}, \ldots, x_{n}\right) \otimes \mathbb{F}_{p}\left[y, \ldots, y_{n}\right] & p \text { odd }\end{cases}
$$


2.5. Restrictions and transfers. Let $H \subset G$ be a subgroup, and note that $E G$ is a contractible space with a free, cellular action of $H$, so that $B H \simeq E G / H$. Hence (cf. 2.3) we have a map

$$
B H=E G / H \rightarrow E G / G=B G .
$$

This induces a restriction map $\operatorname{res}_{H}^{G}: H^{*}(G, M) \rightarrow H^{*}\left(H,\left.M\right|_{H}\right)$. Note that if $R$ is a ring with a trivial action of $G$, then the restriction map is a map of graded $R$-algebras.

Now if we consider the cell structure of $E G$, then over any cell $\sigma$ of $B G$ there are $[G: H]=\#(G / H)=n$ cells $g_{1} \tilde{\sigma}, \ldots, g_{n} \tilde{\sigma}$ of $B H$, where $\tilde{\sigma}$ is some fixed chosen cell above $\sigma$ and $g_{1}, \ldots, g_{n}$ are coset representatives for $G / H$. We define

$$
\psi: C_{*}(B G, \mathbb{Z}) \rightarrow C_{*}(B H, \mathbb{Z})
$$

by setting $\psi(\sigma)=\sum_{i=1}^{n} g_{i} \tilde{\sigma}$. This idea can be exploited in slightly greater generality to construct a transfer map $\operatorname{tr}_{H}^{G}: H^{*}\left(H,\left.M\right|_{H}\right) \rightarrow H^{*}(G, M)$.

There are a few basic formulas involving these maps.

(1) If $H \subset K \subset G$, then $\operatorname{res}_{H}^{K} \cdot \operatorname{res}_{K}^{G}=\operatorname{res}_{H}^{G}$.

(2) $\operatorname{tr}_{H}^{G} \cdot \operatorname{res}_{H}^{G}(x)=[G: H] x, \quad \forall x \in H^{*}(G, M)$.

(3) Suppose that $H$ and $K$ are subgroups of $G$, and that $G$ is written as a disjoint union $\cup_{i=1}^{m} H g_{i} K$ of double cosets. Then

$$
\operatorname{res}_{H}^{G} \cdot \operatorname{tr}_{K}^{G}=\sum_{i=1}^{m} \operatorname{tr}_{H \cap K^{g_{i}}}^{H} \cdot \operatorname{res}_{H \cap K^{g_{i}}}^{K^{g_{i}}} \cdot C_{g_{i}},
$$

where $C_{g_{i}}$ is induced by conjugation with $g_{i}$.

2.6. Exercise. Let $P$ be a Sylow $p$-subgroup of $G$, and $N_{G}(P)$ its normalizer in $G$. If $P$ is abelian, show that restriction induces an isomorphism

$$
H^{*}\left(G, \mathbb{F}_{p}\right) \cong H^{*}\left(N_{G}(P), \mathbb{F}_{p}\right) \text {. }
$$

2.7. Lyndon-Hochschild-Serre spectral sequence. If $H$ is a normal subgroup of $G$, then $G / H$ acts freely on $B H \simeq E G / H$, and so we have a fibration

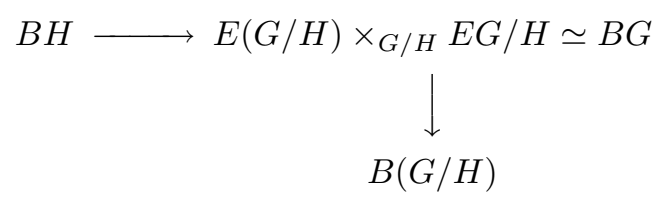

This gives a spectral sequence

$$
E_{2}^{p, q}=H^{p}\left(G / H, H^{q}(H, M)\right) \Rightarrow H^{p+q}(G, M),
$$

in which the $E_{2}$-page involves local coefficient cohomology determined by an action of $G / H$ on $H^{*}(H, M)$.

2.8. ExAmples. (1) Let $G$ be the alternating group $A_{4}$. There is a group extension

$$
1 \rightarrow(\mathbb{Z} / 2)^{2} \rightarrow G=A_{4} \rightarrow \mathbb{Z} / 3 \rightarrow 1 .
$$

The associated mod 2 cohomology spectral sequence collapses to give the formula

$$
\begin{aligned}
H^{*}\left(A_{4}, \mathbb{Z} / 2\right) & \cong \mathbb{F}_{2}\left[x_{1}, x_{3}\right]^{\mathbb{Z} / 3} \\
& \cong \mathbb{F}_{2}\left[u_{2}, v_{3}, w_{3}\right] /\left(u_{2}^{3}+v_{3}^{2}+w_{3}^{2}+v_{3} w_{3}\right) .
\end{aligned}
$$


(2) Let $G$ be the dihedral group $D_{8}$, which can be written as the wreath product $\mathbb{Z} / 2 \imath \mathbb{Z} / 2$ or equivalently as the semidirect product $(\mathbb{Z} / 2 \times \mathbb{Z} / 2) \rtimes \mathbb{Z} / 2$. The $\bmod 2$ cohomology spectral sequence of the semidirect product extension collapses strongly at $E_{2}$ and gives the formula

$$
H^{*}\left(D_{8}, \mathbb{F}_{2}\right) \cong \mathbb{F}_{2}\left[x_{1}, e_{1}, y_{2}\right] /\left(x_{1} e_{1}\right) .
$$

Given any $G$, we can find a monomorphism $G \rightarrow U(n)$ to obtain a fibration

$$
U(n) / G \rightarrow B G \rightarrow B U(n) .
$$

Here $U(n)$ is the unitary group of rank $n$, and we are implicitly referring to the fact that classifying spaces can be constructed not just for finite groups but also for topological groups such as $U(n)$. Recall that $H^{*}\left(B U(n), \mathbb{F}_{p}\right)$ is isomorphic to a polynomial algebra $\mathbb{F}_{p}\left[c_{1}, \ldots, c_{n}\right]$ (the generators are the universal Chern classes). Venkov [38] used the above fibration sequence to prove

2.9. Theorem. If $G$ is a finite subgroup of $U(n)$, then $H^{*}\left(G, \mathbb{F}_{p}\right)$ is a finitely generated module over $H^{*}\left(B U(n), \mathbb{F}_{p}\right)$, and its Poincaré series is a rational function of the form

$$
p_{G}(t)=\sum \operatorname{dim} H^{i}\left(G, \mathbb{F}_{p}\right) t^{i}=\frac{r(t)}{\prod_{i=1}^{m}\left(1-t^{2 i}\right)},
$$

where $r(t) \in \mathbb{Z}[t]$.

2.10. Example. Let $G=Q_{8}$, the quaternion group of order 8. Then $G \subset$ $S U(2)$, and we have a fibration

$$
S U(2) / G \rightarrow B Q_{8} \rightarrow B S U(2) .
$$

Here $H^{*}\left(B S U(2), \mathbb{F}_{2}\right) \cong \mathbb{F}_{2}\left[u_{4}\right]$ and $H^{*}\left(B Q_{8}, \mathbb{F}_{2}\right)$ is a free $\mathbb{F}_{2}\left[u_{4}\right]$-module, with basis given by

$$
H^{*}\left(S U(2) / Q_{8}\right) \cong \mathbb{F}_{2}\left[x_{1}, y_{1}\right] /\left(x_{1}^{2}+x_{1} y_{1}+y_{1}^{2}, x_{1}^{2} y_{1}+x_{1} y_{1}^{2}\right) .
$$

Then $H^{*}\left(Q_{8}\right) /\left(u_{4}\right) \cong H^{*}\left(S U(2) / Q_{8}, \mathbb{F}_{2}\right)$.

Question. What is the order of the pole of $p_{G}(t)$ at $t=1$ ? This is known as the Krull dimension of $H^{*}\left(G, \mathbb{F}_{p}\right)$.

Answer. The order of the pole of $p_{G}(t)$ at $t=1$ is the $p$-rank $r_{p}(G)$ of $G$, defined as

$$
r_{p}(G)=\max \left\{n \mid(\mathbb{Z} / p)^{n} \subset G\right\} .
$$

In the next section, we will try to explain this answer using representation theory.

\section{Minimal resolutions}

Let $P$ be a finite $p$-group, $k$ a field of characteristic $p$, and $M$ a finitely generated $k P$-module. Using the nilpotence of the augmentation ideal $I \subset k P$, one can see that the rank of the projective cover of $M$ is $r=\operatorname{dim} M / I M=\operatorname{dim} H_{0}(P, M)$. Hence we have an exact sequence

$$
0 \rightarrow \Omega^{1}(M) \rightarrow(k P)^{r} \rightarrow M \rightarrow 0
$$

(this sequence defines $\Omega^{1}(M)$ ) where the right hand map induces an isomorphism $H_{0}\left(P,(k P)^{r}\right) \cong\left(\mathbb{F}_{p}\right)^{r} \rightarrow H_{0}(P, M)$. The long exact homology sequence associated to the above short exact sequence shows that $\operatorname{dim} H_{0}\left(P, \Omega^{1}(M)\right)=\operatorname{dim} H_{1}(P, M)$; 
this last number is then in turn the rank of the projective cover of $\Omega^{1}(M)$. Continuing on like this, and identifying

$$
H_{i}(P, M) \cong H^{i}\left(P, M^{*}\right) \quad \text { where } M^{*}=\operatorname{Hom}(M, k),
$$

we obtain the following statement.

3.1. Proposition. If $P_{*} \rightarrow M$ is a minimal projective resolution for $M$, then

$$
\operatorname{dim}_{\mathbb{F}_{p}} P_{i}=|P| \operatorname{dim} H^{i}\left(P, M^{*}\right) .
$$

3.2. Corollary. The following are equivalent:

(1) $M$ is projective,

(2) $H^{r}(P, M)=0$ for some $r>0$, and

(3) $H^{i}(P, M)=0$ for all $i>0$.

We want to determine projectivity by restriction to certain subgroups. We will use group cohomology to do this, by applying the following basic result of Serre $([32])$ :

3.3. THEOREM. Let $P$ denote a finite $p$-group which is not elementary abelian. Then there exist non-zero elements $\gamma_{1}, \ldots, \gamma_{n} \in H^{1}\left(P, \mathbb{F}_{p}\right)$ such that

$$
\beta\left(\gamma_{1}\right) \beta\left(\gamma_{2}\right) \cdots \beta\left(\gamma_{n}\right)=0
$$

where $\beta$ is the Bockstein.

Now if $\gamma \in H^{1}(\mathbb{Z} / p, \mathbb{Z} / p)$ corresponds to the identity homomorphism $\mathbb{Z} / p \rightarrow \mathbb{Z} / p$, then its Bockstein in $H^{2}(\mathbb{Z} / p, \mathbb{Z} / p) \cong \operatorname{Ext}_{\mathbb{F}_{p}[\mathbb{Z} / p]}^{2}(\mathbb{Z} / p, \mathbb{Z} / p)$ is the sequence

$$
\mathbb{F}_{p} \rightarrow \mathbb{F}_{p}[\mathbb{Z} / p] \stackrel{t-1}{\longrightarrow} \mathbb{F}_{p}[\mathbb{Z} / p] \rightarrow \mathbb{F}_{p}
$$

For non-zero $\gamma_{i} \in H^{1}\left(P, \mathbb{F}_{p}\right)$, we take a homomorphism $\phi: P \rightarrow \mathbb{Z} / p$ representing it, with kernel $H_{i} \triangleleft P$ (a maximal subgroup). Pulling back the representative for the Bockstein of the identity map of $\mathbb{Z} / p$ gives an expression for $\beta \gamma_{i}$ as a sequence

$$
\mathbb{F}_{p} \rightarrow \mathbb{F}_{p}\left[P / H_{i}\right] \rightarrow \mathbb{F}_{p}\left[P / H_{i}\right] \rightarrow \mathbb{F}_{p} .
$$

Taking products of cohomology classes corresponds to splicing extensions, so that $\beta\left(\gamma_{1}\right) \cdots \beta\left(\gamma_{n}\right)$ is represented by the sequence

$$
\begin{aligned}
\mathbb{F}_{p} \rightarrow \mathbb{F}_{p}\left[P / H_{n}\right] \rightarrow \mathbb{F}_{p}\left[P / H_{n}\right] & \rightarrow \mathbb{F}_{p}\left[P / H_{n-1}\right] \rightarrow \mathbb{F}_{p}\left[P / H_{n-1}\right] \rightarrow \\
\cdots & \rightarrow \mathbb{F}_{p}\left[P / H_{1}\right] \rightarrow \mathbb{F}_{p}\left[P / H_{1}\right] \rightarrow \mathbb{F}_{p} .
\end{aligned}
$$

We leave off the copies of $\mathbb{F}_{p}$ on either end and interpret this as a cochain complex $C^{*}$, with $H^{*}\left(C^{*}\right)=H^{*}\left(S^{2 n-1}, \mathbb{F}_{p}\right)$.

3.4. Proposition. If $P$ is not elementary abelian, then the $\mathbb{F}_{p}[P]$-module $M$ is projective if and only if $\left.M\right|_{K}$ is projective for each maximal subgroup $K \subset P$.

Proof. Consider $C^{*} \otimes M$; we will compute $H^{*}\left(P, C^{*} \otimes M\right)$ in two different ways (what we are computing is sometimes called the hypercohomology of $P$ with coefficients in $\left.C^{*} \otimes M\right)$. There are two spectral sequences

(1) $E_{2}^{r, q}=H^{r}\left(P, H^{q}\left(C^{*} \otimes M\right)\right) \Rightarrow H^{r+q}\left(P, C^{*} \otimes M\right)$, and

(2) $E_{1}^{r, q}=H^{q}\left(P, C^{r} \otimes M\right) \Rightarrow H^{r+q}\left(P, C^{*} \otimes M\right)$. 
The first one has a single differential determined by $\beta\left(\gamma_{1}\right) \cdots \beta\left(\gamma_{n}\right)=0$ (by construction), and so it collapses at $E_{2}$ giving

$$
H^{\ell}\left(P, C^{*} \otimes M\right) \cong H^{\ell}(P, M) \oplus H^{\ell-2 n+1}(P, M) .
$$

For the second spectral sequence, note that

$$
H^{q}\left(P, C^{r} \otimes M\right) \cong H^{q}\left(H_{i}, M\right),
$$

where $H_{i} \subset P$ is maximal. So if $\left.M\right|_{K}$ is projective for any maximal $K \subset P$, we get that $H^{t}\left(P, C^{*} \otimes M\right)$ vanishes for $t \gg 0$. Combining these two calculations shows that $H^{t}(P, M)=0$ for $t \gg 0$, and so $M$ is projective. The opposite implication is clear.

An immediate consequence is

3.5. Theorem. (Chouinard) An $\mathbb{F}_{p}[G]$-module $M$ is projective if and only if $\left.M\right|_{E}$ is projective for all elementary abelian p-subgroups of $G$.

Proof. Let $P \in \operatorname{Syl}_{p}(G)$; if $\left.M\right|_{P}$ is free (equivalently, projective), then so is the module $\mathbb{F}_{p}[G] \otimes_{\mathbb{F}_{p}[P]} M$; as this induced module contains $M$ as a direct summand (use the fact that the index of $P$ in $G$ is prime to $p$ ), it follows that $M$ is projective. This shows that $M$ is projective if and only if $\left.M\right|_{P}$ is projective; now apply our previous result repeatedly to reduce the problem of testing projectivity for $M$ to the problem of examining $\left.M\right|_{E}$ for every elementary abelian subgroup of $P$.

If $V_{*}$ is a graded $k$-vector space, we can define its growth rate $\gamma\left(V_{*}\right)$ by

$$
\gamma\left(V_{*}\right)=\min \left\{n \geq 0 \mid \lim _{t \rightarrow \infty} \frac{\operatorname{dim} V_{t}}{t^{n}}=0\right\} .
$$

3.6. Definition. The complexity $\operatorname{cx}_{G}(M)$ of a $k G$-module $M$ is defined to be $\gamma\left(k \otimes_{k G} P_{*}\right)$, where $P_{*}$ is a minimal projective resolution of $M$ over $k G$.

3.7. Theorem. (Quillen/Alperin-Evens [28], [7])

$$
\operatorname{cx}_{G}(M)=\max _{E \subset G}\left\{\operatorname{cx}_{E}\left(\left.M\right|_{E}\right)\right\},
$$

where $E$ runs through elementary abelian p-subgroups of $M$.

We can sketch a proof of this: as before we can reduce it to $p$-groups. Now for a $p$-group $P$ we have that

$$
\begin{aligned}
\operatorname{cx}_{P}(M) & =\gamma\left(H^{*}\left(P, M^{*}\right)\right) \\
& =\max _{E} \gamma\left(H^{*}\left(E, M^{*}\right)\right) \\
& =\max _{E} \operatorname{cx}_{E}(M) .
\end{aligned}
$$

Going from the first to second line here uses our previous argument and Serre's result.

For trivial coefficients, this implies that the Krull dimension of $H^{*}\left(G, \mathbb{F}_{p}\right)$ is precisely $r_{p}(G)$, as we have an explicit computation of $H^{*}\left(E, \mathbb{F}_{p}\right)$.

Let $V_{G}(k)$ denote the maximal ideal spectrum for $H^{*}(G, k)$. The restriction $\operatorname{res}_{E}^{G}$ induces a map

$$
\left(\operatorname{res}_{E}^{G}\right)^{*}: V_{E}(k) \rightarrow V_{G}(k) .
$$


3.8. Theorem. (Quillen $[\mathbf{2 8}]$ ) Let $A_{p}(G)$ be the set of all elementary abelian p-subgroups of $G$. Then

$$
V_{G}(k)=\cup_{E \in A_{p}(G)}\left(\operatorname{res}_{E}^{G}\right)^{*}\left(V_{E}(k)\right) .
$$

We can view a maximal ideal $\mathfrak{m} \in V_{G}(k)$ as the kernel of a nonzero homomorphism $H^{*}(G, k) \stackrel{\alpha}{\rightarrow} \bar{k}$, where $\bar{k}$ is an algebraic closure of $k$. Quillen's theorem says that every such homomorphism $\alpha$ is of the form

$$
H^{*}(G, k) \stackrel{\operatorname{res}_{E}^{G}}{\longrightarrow} H^{*}(E, k) \stackrel{\beta}{\longrightarrow} \bar{k}
$$

for some $E \in A_{p}(G)$.

For more details on the methods outlined in this section, we refer the reader to the paper by J. Carlson [15].

\section{Computations and further structure}

Let $\mathcal{F}$ denote a family of subgroups of $G$, i.e., if $H \in \mathcal{F}, H^{\prime} \subset H$, then $H^{\prime} \in \mathcal{F}$ and $g H g^{-1} \in \mathcal{F}$ for any $g \in G$. Then we can define

$$
\lim _{H \in \mathcal{F}} H^{*}\left(H, \mathbb{F}_{p}\right)=\left\{\left(\alpha_{H}\right) \text { such that } \begin{array}{l}
\alpha_{H^{\prime}}=\operatorname{res}_{H^{\prime}}^{H} \alpha_{H} \text { if } H^{\prime} \subset H \\
\alpha_{H^{\prime}}=c_{g} \alpha_{H} \text { if } H^{\prime}=g H g^{-1}
\end{array}\right\}
$$

We can use this construction to reinterpret our previous results.

4.1. Theorem. (Cartan-Eilenberg $[\mathbf{2 0}]$ ) Let $S_{p}(G)$ denote the family of all p-subgroups of $G$. Then the restrictions induce an isomorphism

$$
H^{*}\left(G, \mathbb{F}_{p}\right) \cong \lim _{P \in S_{p}(G)} H^{*}\left(P, \mathbb{F}_{p}\right) .
$$

4.2. Theorem. (Quillen-Venkov $[\mathbf{3 1}]$ ) Let $A_{p}(G)$ denote the family of all $p$ elementary abelian subgroups of $G$. Then the restrictions induce an $F$-isomorphism ${ }^{1}$

$$
\theta: H^{*}\left(G, \mathbb{F}_{p}\right) \rightarrow \lim _{E \in A_{p}(G)} H^{*}\left(E, \mathbb{F}_{p}\right) .
$$

We need to compute $H^{*}\left(G, \mathbb{F}_{p}\right)$ for interesting classes of groups. In many cases this will involve finding a collection of subgroups $H_{1}, \ldots, H_{\ell}$ such that the map

$$
H^{*}(G) \rightarrow \oplus_{i=1}^{\ell} H^{*}\left(H_{i}\right)
$$

is injective, in which case this collection is said to detect the cohomology.

4.3. Calculational methods. Calculating the cohomology of finite groups can be quite challenging, as it will involve a number of complicated ingredients. We outline the main techniques and provide some examples.

How to compute:

Step 1. Reduce to the Sylow $p$-subgroup via the Cartan-Eilenberg result, and then combine information about the cohomology of $p$-groups with stability conditions.

Step 2. Determine $A_{p}(G)$ and use Quillen's result to compute

$$
H^{*}(G) / \sqrt{O}
$$

\footnotetext{
${ }^{1}$ By which we mean that the kernel of $\theta$ is nilpotent and that a sufficiently high power of any element in the target lies in the image of $\theta$.
} 
where $\sqrt{O}$ is the radical of the ideal of elements of strictly positive degree. This will require computing rings of invariants of the form $H^{*}(E)^{N}$, where $E$ is $p$-elementary abelian and $N$ is the normalizer of $E$.

Step 3. Let $\left|A_{p}(G)\right|$ be the $G$-CW complex which realizes the poset $A_{p}(G)$. A result due to Webb [39] implies that $H^{*}\left(G, \mathbb{F}_{p}\right)$ can be computed from the cohomology of the groups $N_{G}(E)$ and the cohomology of certain of their intersections. These are the intersections which appear as simplex stabilizers for the action of $G$ on $\left|A_{p}(G)\right|$.) More precisely, the mod $p$ Leray spectral sequence associated to the projection $E G \times_{G}\left|A_{p}(G)\right| \rightarrow\left|A_{p}(G)\right| / G$ collapses at $E_{2}$ with

$$
E_{2}^{s, t}= \begin{cases}H^{t}(G) & s=0 \\ 0 & s>0\end{cases}
$$

If $G$ is of Lie type, we can use the Tits building and information about the parabolic subgroups of $G$ to facilitate this calculation, as it will be equivariantly equivalent to the poset space above.

4.4. Example. Let $S_{n}$ be the symmetric group of degree $n$. In this case we have that Quillen's map induces a mod 2 isomorphism

$$
H^{*}\left(S_{n}, \mathbb{F}_{2}\right) \stackrel{\cong}{=} \lim _{E \in A_{2}\left(S_{n}\right)} H^{*}\left(E, \mathbb{F}_{2}\right) .
$$

This means that the cohomology is detected on elementary abelian subgroups. To determine $H^{*}\left(S_{n}\right)$ we need to glue together the different bits from these detecting subgroups. This can be done for a general $S_{n}$, using invariant theory and combinatorics. In fact, according to Milgram [3] and Feshbach $[\mathbf{2 2}], H^{*}\left(S_{\infty}\right)$ surjects onto $H^{*}\left(S_{n}\right)$, and $H^{*}\left(S_{\infty}\right)$ is known (from Nakaoka [27]). As an explicit example, $H^{*}\left(S_{4}\right) \cong \mathbb{F}_{2}\left[x_{1}, y_{2}, c_{3}\right] /\left(x_{1} c_{3}\right)$, is detected by a map to

$$
H^{*}\left(S_{4}\right) \rightarrow H^{*}\left(V_{1}\right)^{\mathbb{Z} / 2} \cong \mathbb{F}_{2}\left[\sigma_{1}, \sigma_{2}\right] \quad \oplus \quad H^{*}\left(V_{2}\right)^{G L_{2}\left(\mathbb{F}_{2}\right)} \cong \mathbb{F}_{2}\left[d_{2}, d_{3}\right]
$$

where

$$
\begin{aligned}
& x_{1} \mapsto\left(\sigma_{1}, 0\right) \\
& y_{2} \mapsto\left(\sigma_{2}, d_{2}\right) \\
& c_{3} \mapsto\left(0, d_{3}\right)
\end{aligned}
$$

4.5. Remarks. (1) $H^{*}\left(A_{n}, \mathbb{F}_{2}\right)$ can be obtained from the cohomology of $H^{*}\left(S_{n}, \mathbb{F}_{2}\right)$.

(2) If $G$ is one of the standard algebraic groups over $\mathbb{Z}$, then $H^{*}\left(G\left(\mathbb{F}_{q}\right), \mathbb{F}_{p}\right), p \neq q$, was computed by Quillen $[\mathbf{2 9}]$ and others. In these cases, the cohomology is detected on abelian subgroups.

(3) Quillen [30] also computed the mod 2 cohomology of the extra-special 2-groups, described as central extensions

$$
1 \rightarrow \mathbb{Z} / 2 \rightarrow P \rightarrow(\mathbb{Z} / 2)^{r} \rightarrow 1 .
$$

This can be done with the Eilenberg-Moore spectral sequence.

We now give some examples of calculations for simple groups at $p=2$.

4.6. ExAmples. (1) $G=L_{3}(2)$ : we use the Tits building, which is equivalent to $\left|A_{2}(G)\right|$; in this case it has quotient

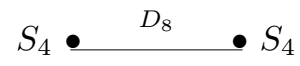


We compute the cohomology of $G$ as the intersection of two copies of the cohomology of $S_{4}$ in the cohomology of $D_{8}$ :

$$
H^{*}\left(L_{3}(2)\right) \cong \mathbb{F}_{2}\left[u_{2}, v_{3}, w_{3}\right] /\left(v_{3} w_{3}\right) .
$$

(2) $G=A_{6}$ : here we use $H^{*}\left(S_{6}\right) /\left(\sigma_{1}\right)=H^{*}\left(A_{6}\right)$, yielding

$$
H^{*}\left(A_{6}\right)=\mathbb{F}_{2}\left[\sigma_{2}, \sigma_{3}, c_{3}\right] /\left(c_{3} \sigma_{3}\right) .
$$

(3) $G=M_{11}$, the first Mathieu group; in this case $\left|A_{2}(G)\right| / G$ looks like:

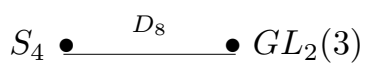

giving

$$
H^{*}\left(M_{11}\right) \cong \mathbb{F}_{2}\left[v_{3}, u_{4}, w_{5}\right] /\left(w_{5}^{2}+v_{3}^{2} u_{4}\right) .
$$

(4) (Adem-Milgram [4]) If $S$ is a simple group of rank 3 or less at $p=2$, then $H^{*}\left(S, \mathbb{F}_{2}\right)$ is Cohen-Macaulay. This means that the cohomology ring is a finitely generated free module over a polynomial subring.

From the calculation above, we see that the mod 2 cohomologies of $A_{6}$ and $L_{3}(2)$ are isomorphic, even though there is no non-trivial homomorphism between these two simple groups. However, there is an infinite amalgam $\Gamma=\Sigma_{4} *_{D_{8}} \Sigma_{4}$ such that there are homomorphisms $\Gamma \rightarrow A_{6}$ and $\Gamma \rightarrow L_{3}(2)$ inducing mod 2 homology equivalences. From this one can show that the classifying spaces $B A_{6}$ and $B L_{3}(2)$ are equivalent at $p=2$.

4.7. Depth and detection. An important invariant for the $\bmod p$ cohomology $H^{*}\left(G, \mathbb{F}_{p}\right)$ is its depth, defined as the maximal length of a regular sequence. We have the following basic result of Duflot [21].

4.8. TheOREM. If $Z(G)$ is the center of $G$ and $r_{p}(Z(G))=z$, then

$$
\operatorname{depth} H^{*}(G) \geq z \text {. }
$$

We will sketch a proof of this, for a $p$-group $P$. Let $Z(P) \cong \mathbb{Z} / p^{n_{1}} \times \cdots \times \mathbb{Z} / p^{n_{z}}$, and for each summand choose a 1-dimensional faithful complex representation $\chi_{i}$; this extends to a representation of $Z(P)$. Let $V_{i}=\operatorname{Ind}_{Z(P)}^{P}\left(\chi_{i}\right)$ and consider its associated sphere $S\left(V_{i}\right)$. Then, if $X=S\left(V_{1}\right) \times \cdots \times S\left(V_{z}\right), X$ has an action of $P$. Consider the $\bmod p$ Serre spectral sequence for the fibration

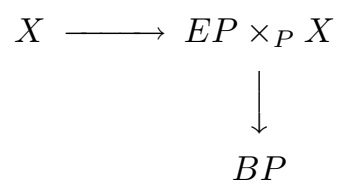

Let $s=2[P: Z]$. Then $H^{*}(X) \cong \Lambda_{\mathbb{F}_{p}}\left(u_{1}, \ldots, u_{z}\right)$, where the degree of $u_{i}$ is $s-1$; each class $u_{i}$ is invariant under the action of $P$ on $H^{*}(X)$ and so represents a class in $E_{2}^{0, s-1}$. For positional reasons $u_{i}$ survives to $E_{s}^{0, s-1}$, and we write $d^{s}\left(u_{i}\right)=\alpha_{i} \in$ $H^{s}(P)$. The key result which can be proved inductively is that $\alpha_{1}, \ldots, \alpha_{z}$ form a regular sequence. This implies that there is an isomorphism

$$
H^{*}\left(E P \times_{P} X\right) \cong H^{*}(P) /\left(\alpha_{1}, \ldots, \alpha_{z}\right) .
$$

There is a special case: if $r_{p}(Z(P))=r_{p}(P)$, then every element of order $p$ in $P$ is central. In this case

- $H^{*}(P)$ is Cohen-Macaulay, being free and finitely generated over $\mathbb{F}_{p}\left[\alpha_{1}, \ldots, \alpha_{z}\right]$. 
- $P$ acts freely on $X$ and there is an isomorphism

$$
H^{*}(X / P) \cong H^{*}(P) /\left(\alpha_{1}, \ldots, \alpha_{z}\right) .
$$

Note that we have a special geometric orientation class

$$
\mu \in H^{\text {top }}(X / P) .
$$

This element can be pulled back and used to construct an "undetectable" class in $H^{*}(P)$, yielding

4.9. Theorem. (Adem-Karagueuzian [2]) If every element of order $p$ in $P$ is central (i.e., $P$ is a $p C$-group), then $H^{*}(P)$ is Cohen-Macaulay and $H^{*}(P)$ is undetectable: there exists an element $x \neq 0$ in $H^{*}(P)$ such that $\operatorname{res}_{H}^{P}(x)=0$ for all proper subgroups $H \subset P$.

On the other hand, we have

4.10. Theorem. (Carlson $[\mathbf{1 4}])$ If $H^{*}\left(G, \mathbb{F}_{p}\right)$ has depth $r$, then this cohomology is detected by subgroups of the form $C_{G}(E)$, where $E \subset G$ is p-elementary abelian of rank $r$.

4.11. REMARKs. In particular, this tells us that if $H^{*}\left(G, \mathbb{F}_{p}\right)$ is Cohen-Macaulay, then it is detected on subgroups of the form $C_{G}(E)$, where $E$ has maximal rank. These are necessarily $p \mathrm{C}$-groups. Hence we have the converse statement, that if $H^{*}\left(G, \mathbb{F}_{p}\right)$ is Cohen-Macaulay and undetectable, then $G$ is a $p \mathrm{C}$-group. We can think of $p \mathrm{C}$-groups as "universal detectors" for Cohen-Macaulay cohomology rings. Hence determining their cohomology is a basic problem.

4.12. ExAmple. Consider a "universal" or maximal central extension

$$
1 \rightarrow H_{2} E \rightarrow P \rightarrow E \rightarrow 1
$$

where $E=(\mathbb{Z} / p)^{n}, p$ is an odd prime, and the differential $H^{1}\left(H_{2}(E)\right) \rightarrow H^{2}(E)$ is an isomorphism. Then one can show that if $p>\left(\begin{array}{l}n \\ 2\end{array}\right)+1$, the Serre spectral sequence collapses at $E_{3}$ and we have an exact sequence

$$
0 \rightarrow\left(\zeta_{1}, \ldots, \zeta_{\left(\begin{array}{c}
n+1 \\
2
\end{array}\right)}\right) \rightarrow H^{*}(P) \rightarrow \operatorname{Tor}_{\mathbb{F}_{p}\left[c_{i j}\right]}\left(\Lambda\left(e_{1}, \ldots, e_{n}\right), \mathbb{F}_{p}\right) \rightarrow 0
$$

where the Tor term is determined by $c_{i j} \mapsto e_{i} e_{j}$ for $i<j, i, j=1, \ldots, n$ (see [5]).

4.13. Problem. Motivated by the above example, we can raise the following question. Let

$$
1 \rightarrow V \rightarrow P \rightarrow W \rightarrow 1
$$

be a central extension, where $V$ and $W$ are elementary abelian groups. Can the Eilenberg-Moore spectral sequence fail to collapse at

$$
E_{2}=\operatorname{Tor}_{H *(K(V, 2))}\left(H^{*}(W), \mathbb{F}_{p}\right) ?
$$

If so, give reasonable conditions on the $k$-invariants which imply a collapse.

4.14. Duality for group cohomology. We briefly recall an important condition on $H^{*}\left(G, \mathbb{F}_{p}\right)$, related to duality. Let $k\left[\zeta_{1}, \ldots, \zeta_{r}\right] \subset H^{*}(G, k)$ be a homogeneous system of parameters having $\operatorname{deg} \zeta_{i}=n_{i} \geq 2$. 
4.15. TheOrem. (Benson-Carlson [11]) There exists a finite complex $C$ of projective $k G-$ modules with $H^{*}\left(\operatorname{Hom}_{k}(C, k)\right) \cong \Lambda\left(\bar{\zeta}_{1}, \ldots, \bar{\zeta}_{r}\right)$, with $\operatorname{deg} \bar{\zeta}_{i}=n_{i}-1$. There is a spectral sequence with

$$
E_{2}^{*, *}=H^{*}(G, k) \otimes \Lambda\left(\bar{\zeta}_{1}, \ldots, \bar{\zeta}_{r}\right)
$$

converging to $H^{*}\left(\operatorname{Hom}_{k G}(C, k)\right.$, which satisfies Poincaré duality in formal dimension $s=\sum_{i-1}^{r}\left(n_{i}-1\right)$. In this spectral sequence we have $d_{n_{i}}\left(\bar{\zeta}_{i}\right)=\zeta_{i}$, and if $H^{*}(G, k)$ is Cohen-Macaulay, then

$$
H^{*}(G, k) /\left(\zeta_{1}, \ldots, \zeta_{r}\right) \cong H^{*}\left(\operatorname{Hom}_{k G}(C, k)\right) .
$$

More succinctly,

4.16. Theorem. Suppose that $G$ is a finite group. If the ring $H^{*}(G, k)$ is Cohen-Macaulay, then it is Gorenstein with a-invariant zero.

4.17. Remarks. In the Cohen-Macaulay case, $H^{*}(G) /\left(\zeta_{1}, \ldots, \zeta_{r}\right)$ satisfies Poincaré duality, and its top degree is $\sum_{i=1}^{r}\left(n_{i}-1\right)=d$. The " $a$-invariant" is computed as $a=d-\sum_{i=1}^{r}\left(\left|\zeta_{i}\right|-1\right)=0$ in this case. Note the functional equation: if $p_{G}(t)$ is the Poincaré series for $H^{*}(G)$, then

$$
p_{G}(1 / t)=(-t)^{r} p_{G}(t) .
$$

The previous theorem asserts that if $G$ is a finite group of rank $r$, then there exists a projective $\mathbb{Z} G$ chain complex $C$ with

$$
H^{*} C^{*} \cong H^{*}\left(S^{n_{1}-1} \times \cdots \times S^{n_{r}-1}\right) .
$$

In the next section we will describe the analogous problem in a geometric setting, i.e., using free group actions on products of spheres. This turns out to be much more difficult, as we shall see.

\section{Cohomology and actions of finite groups}

We start by recalling a basic result.

5.1. Theorem. (P. A. Smith, 1940 [33]) If a finite group $G$ acts freely on a sphere, then all of its abelian subgroups are cyclic.

What this means is that $G$ does not contain any subgroup of the form $\mathbb{Z} / p \times \mathbb{Z} / p$ ("the $p^{2}$ condition").

5.2. ExAmples. (1) $\mathbb{Z} / n$ acts freely on any $S^{2 k+1}$.

(2) The quaternion group $Q_{8}$ acts freely on $S^{3}$.

Later, Milnor [26] proved

5.3. THEOREM. If a finite group $G$ acts freely on $S^{n}$. then every involution in $G$ is central.

For example, the dihedral group $D_{2 p}$ cannot act freely on any sphere ("the $2 p$ condition"). However, it turns out that this is really a geometric condition, as we have

5.4. TheOrem. (Swan [36]) A finite group $G$ acts freely on a finite complex $X \simeq S^{n}$ if and only if every abelian subgroup of $G$ is cyclic.

Finally, using surgery theory, it was shown that 
5.5. TheOrEm. (Madsen-Thomas-Wall $[\mathbf{2 5}]) G$ acts freely on some sphere if and only if $G$ satisfies both the $p^{2}$ and the $2 p$ condition for all primes $p$.

If $G$ acts freely on $X=S^{n}$, preserving orientation, we have a Gysin sequence (with $\mathbb{Z}$ or $\mathbb{F}_{p}$ coefficients):

$$
\cdots \rightarrow H^{i+n}(X / G) \rightarrow H^{i}(G) \stackrel{\cup x}{\longrightarrow} H^{i+n+1}(G) \rightarrow H^{i+n+1}(X / G) \rightarrow \cdots
$$

where $x \in H^{n+1}(G)$ is the Euler class of the action. Note that $H^{s}(X / G)=0$ for $s>n$; this implies that the map

$$
\cup x: H^{i}(G) \rightarrow H^{i+n+1}(G)
$$

is an isomorphism for all $i>0$. This in turn implies that $G$ has periodic cohomology, i.e., $H^{*}\left(G, \mathbb{F}_{p}\right)$ has Krull dimension one for all $p|| G \mid$. It follows that $G$ satisfies the $p^{2}$-condition for all primes $p$.

5.6. Remarks. (1) In fact, $G$ has periodic cohomology if and only if every abelian subgroup in $G$ is cyclic (Artin-Tate [8]).

(2) Given $G$ with periodic cohomology, say of minimal period $d$, acting freely on $X \simeq S^{n}$, then $d \mid n+1$, but $d$ is not necessarily equal to $n+1$.

5.7. ExAmple. Let $S=Z / 3 \rtimes Q_{16}$, where the element of order 8 in $Q_{16}$ acts nontrivially on $\mathbb{Z} / 3$. Then $S$ has period four but does not act freely on any finite homotopy 3 -sphere. Hence there is no closed 3 -manifold $M$ with $\pi_{1}(M) \cong S$.

5.8. ExAmples. Linear spheres. Let $V$ be a unitary or orthogonal representation of $G$ which is fixed-point-free. Then $G$ will act freely on $X=S(V)$. These groups have been characterized by Wolf $[\mathbf{4 0}]$ :

5.9. TheOREM. The group $G$ will act freely on some $S(V)$ if and only if

(1) every subgroup of order $p q$ ( $p, q$ prime) in $G$ is cyclic, and

(2) $G$ does not contain $S L_{2}\left(\mathbb{F}_{p}\right)$ with $p>5$ as a subgroup.

5.10. General restrictions. Consider a closed oriented manifold $M^{n}$ with a free $G$-action preserving orientation. Then $M \rightarrow M / G$ has degree $|G|$. Using the spectral sequence for the fibration $M \rightarrow M / G \rightarrow B G$, one can prove

5.11. ThEOREM. (Browder [12]) If $G$ acts freely on $M^{n}$, preserving orientation, then $|G|$ divides the product

$$
\prod_{s=1}^{n} \operatorname{exponent} H^{s+1}\left(G, H^{n-s}(M, \mathbb{Z})\right) .
$$

In the statement above, note that the cohomology groups which appear are all finite abelian groups, and the exponent is simply the smallest positive integer that annihilates every element in the group.

Consequences. (1) If $M^{n}$ has a free action of $(\mathbb{Z} / p)^{r}$ which is trivial in homology, then the total number of dimensions $0 \leq j<n$ such that $H^{j}\left(M, \mathbb{Z}_{(p)}\right) \neq 0$ must be at least $r$. This follows from the fact that $p \cdot \bar{H}^{*}\left((\mathbb{Z} / p)^{r}, \mathbb{Z}\right)=0$.

(2) If $(\mathbb{Z} / p)^{r}$ acts freely and homologically trivially on $M=\left(S^{n}\right)^{k}$, then $r \leq k$ (Carlsson $[\mathbf{1 6}],[\mathbf{1 7}])$. 
With the help of Tate cohomology, these results can be extended to finite, connected, free $G-\mathrm{CW}$ complexes. The most general conjecture is given by

5.12. Conjecture. (Carlsson $[\mathbf{1 8}],[\mathbf{1 9}])$ If $(\mathbb{Z} / p)^{r}$ acts freely on $X$, then

$$
2^{r} \leq \sum_{i=0}^{\operatorname{dim} X} \operatorname{dim}_{\mathbb{F}_{p}} H_{i}\left(X, \mathbb{F}_{p}\right)
$$

AnCIENT Open PRoBlem. If $(\mathbb{Z} / p)^{r}$ acts freely on $X \simeq S^{n_{1}} \times \cdots S^{n_{k}}$, then is $r \leq k ?$

$$
\begin{aligned}
& \text { Let } \begin{aligned}
r(G) & =\max _{p|| G \mid} r_{p}(G) \\
h(G) & =\min \left\{k \mid G \text { acts freely on a finite } X \simeq S^{n_{1}} \times \cdots \times S^{n_{k}}\right\} .
\end{aligned}
\end{aligned}
$$

then the following conjecture formulated by Benson and Carlson (see [10]) is rather intriguing:

$$
\text { Conjecture: } r(G)=h(G)
$$

Swan's Theorem implies this result for $r(G)=1$. Note that every $G$ acts freely on some product of spheres, so $h(G)$ is well-defined.

5.13. Case study : $S^{n} \times S^{m}$.

Problem. Characterize those finite groups which act freely on a product of two spheres.

We will use representations and bundle theory to address this problem, following the work of Adem, Davis and Ünlü [1].

5.14. Definition. Let $G \subset U(n)$. We define the fixity of this representation as the smallest integer $f$ such that $G$ acts freely on

$$
U(n) / U(n-f-1) \text {. }
$$

Note that $G$ has fixity zero if and only if $G$ acts freely on $S^{2 n-1}$, or equivalently if and only if $S^{2 n-1} / G$ is a (complex) linear space form.

There is a fibration sequence

$$
U(n-f) / U(n-f-1) \rightarrow U(n) / U(n-f-1) \rightarrow U(n) / U(n-f)
$$

in which the map from the total space to the base is a $G$-map.

5.15. TheOREM. If $G \subset U(n)$ has fixity one, then $G$ acts freely and smoothly on $X=S^{2 n-1} \times S^{4 n-5}$.

Proof. Consider the bundle

$$
U(n-1) / U(n-2) \rightarrow U(n) / U(n-2) \rightarrow U(n) / U(n-1) .
$$

It is the associated spherical bundle of a $G$-vector bundle $\xi$ such that $\xi \oplus \xi$ is trivial. Hence $S(\xi \oplus \xi)$ splits (non-equivariantly) as the indicated product of spheres, and has a free $G$-action.

5.16. Corollary. If $G \subset S U(3)$ is a finite subgroup, then $G$ acts freely and smoothly on $S^{5} \times S^{7}$; the finite subgroups of $S U(3)$ include $A_{5}, S L_{3}\left(\mathbb{F}_{2}\right)$, and $3 \cdot A_{6}$.

We now focus on $p$-groups. 
5.17. TheOREM. If $p \geq 3$ is a prime, then a finite $p$-group $P$ acts freely and smoothly on some $S^{n} \times S^{m}$ if and only if $P$ does not contain $\mathbb{Z} / p \times \mathbb{Z} / p \times \mathbb{Z} / p$ as a subgroup.

Sketch of PROOF. The "only if" statement has been known for 50 years $[\mathbf{2 3}]$. For $p>3$, one can show that either $P$ acts freely on some $S(V) \times S(W)$ for some representations $V$ and $W$, or else that there is a representation $P \subset U(p)$ of fixity one, whence $P$ acts freely on $S^{2 p-1} \times S^{4 p-5}$. This result involves using a detailed description of rank two $p$-groups.

Partial results in the case $p=2$ are due to Ünlü [37]. There are 396 2-groups $P$ of order dividing 256 and such that $r_{2}(P)=2$. Of these only one is not yet known to act freely and smoothly on some $S^{n} \times S^{m}$.

More generally, we have the

5.18. Theorem. (The Propagation Theorem) Let $G \subset U(n)$ be such that $G$ acts freely on $U(n) / U(k)$ for some $k>1$. Then, if

$$
(|G|,(n-1) !)=1,
$$

$G$ will act freely and smoothly on

$$
M=S^{2 n-1} \times S^{2 n-3} \times \cdots \times S^{2 k+1} .
$$

5.19. Corollary. Let $P$ be a finite p-group with

- cyclic center,

- a maximal subgroup which is abelian, and

- $\operatorname{rank} f+1<p$.

Then $P$ acts freely and smoothly on

$$
M=S^{2 p-1} \times \cdots \times S^{2(p-f)-1},
$$

i.e., on a product of $f+1$ spheres.

5.20. Example. Suppose that $P$ is an extra-special $p$-group of order $p^{3}$ and exponent $p$. Then $P$ acts freely and smoothly on $S^{2 p-1} \times S^{2 p-3}$.

5.21. Homotopy actions. The preceding results are explicit, geometric examples of a more general homotopy-theoretic construction. The key ingredient is the notion of a space with periodic cohomology, which we now define. The results here are based on the paper by Adem and Smith [6].

5.22. Definition. A space $X$ is said to have periodic cohomology if there exists a cohomology class $\alpha \in H^{*}(X, \mathbb{Z}),|\alpha|>0$, and an integer $d \geq 0$ such that for all coefficient systems $M$ the cup product map

$$
\cup \alpha: H^{n}(X, M) \rightarrow H^{n+|\alpha|}(X, M)
$$

is an isomorphism for $n \geq d$.

5.23. Examples. (1) If $G$ is a finite group, then $B G$ has periodic cohomology if and only if every abelian subgroup in $G$ is cyclic. Indeed, in this case periodicity for trivial coefficients implies periodicity for all coefficients.

(2) If $\Gamma$ is a discrete group of virtually finite cohomological dimension, then $B \Gamma$ has periodic cohomology if and only if every finite subgroup of $\Gamma$ has periodic cohomology. 
5.24. Theorem. (Adem-Smith) A connected $C W$-complex $X$ has periodic cohomology if and only if there exists an orientable spherical fibration

$$
S^{N} \rightarrow E \rightarrow X
$$

such that $E$ has the homotopy type of a finite dimensional complex.

This has the following result as a corollary.

5.25. Corollary. If $Y$ is a simply-connected, finite $G-C W$ complex such that all of its isotropy subgroups have periodic cohomology, then there exists a finite free $G-C W$ complex

for some $N \gg 0$.

$$
X \simeq S^{N} \times Y
$$

5.26. Remarks. Given $G \subset U(n)$ of fixity $f$, we have a geometric realization of our spherical fibration

$$
U(n-f) / U(n-f-1) \rightarrow U(n) / U(n-f-1) \rightarrow U(n) / U(n-f) .
$$

Using the corollary, we have reduced the problem of constructing free $G$-actions on a finite complex $X \simeq S^{n} \times S^{m}$ to the problem of constructing an action of $G$ on a sphere with rank one isotropy subgroups.

5.27. ExAmples. (1) Every rank two $p$-group $P$ acts on an $S(V)$ with rank one isotropy, hence $P$ acts freely on some finite $X \simeq S^{n} \times S^{m}$.

(2) If $S$ is a simple group of rank equal to two different from $P S L_{3}\left(\mathbb{F}_{p}\right)$, then $S$ acts freely on some finite $X \simeq S^{n} \times S^{m}$.

Let $T_{p}=(\mathbb{Z} / p \times \mathbb{Z} / p) \rtimes S L_{2}\left(\mathbb{F}_{p}\right)$. Then one can show that if $T_{p}$ acts on $X=S^{n}$, and $p$ is odd, it has a rank two isotropy subgroup. The equivariant cohomology $H^{*}\left(E T_{p} \times_{T_{p}} X, \mathbb{F}_{p}\right)$ has Krull dimension equal to two.

Problem. Does $T_{p}$ act freely on some $S^{n} \times S^{m}$ ?

Recently, M. Jackson [24] has announced

5.28. TheOREM. A rank 2 group $G$ acts on $Y \simeq S^{N}$ with rank one isotropy if and only if $G$ does not contain $T_{p}$ as a subgroup for any odd prime $p$. Consequently, all such groups act freely on a finite complex $X \simeq S^{n} \times S^{m}$.

Hence we conclude that if $G$ is a rank 2 group not containing any $T_{p}$ as a subgroup ( $p$ odd), then $r(G)=h(G)=2$.

We will provide a more direct proof of this statement for groups of odd order by using some elementary group theory. Applying the local methods in [6], page 433 , it suffices to prove that for each prime $p$ such that $r_{p}(G)=2, G$ acts on a sphere such that the isotropy subgroups have $p$-rank equal to one.

5.29. TheOREM. If $G$ is a finite group of odd order and its rank is equal to two, then for every prime $p$ such that $r_{p}(G)=2$, there exists a $G$-representation $W_{p}$ such that the action of $G$ on $S\left(W_{p}\right)$ has isotropy subgroups having p-rank equal to one.

Proof. First we need some group theory. It is known that every rank two group of odd order is solvable and has a nilpotent commutator subgroup (see [35], page 371). From this it follows easily that for any prime $p$ dividing $|G|$, there exists a $p^{\prime}$ normal subgroup $N$ such that $G^{\prime}=G / N$ has a normal $p$-Sylow subgroup. 
Now let $G_{p}$ denote $S y l_{p}(G)=S y l_{p}\left(G^{\prime}\right)$, and assume that $r_{p}(G)=2$. As in Theorem 3.8, we can find a representation $V_{p}$ for $G_{p}$ such that the $G_{p}$ action has rank one isotropy (indeed, a central element of order $p$ acts freely on $V_{p}$ by construction). Now we can induce this representation up to $G^{\prime}$, to obtain $W_{p}$; the associated sphere $S\left(W_{p}\right)$ will have an action of $G^{\prime}$ and hence of $G$ via $G \rightarrow G^{\prime}$ such that the isotropy subgroups have $p$-rank equal to one.

Hence we have

5.30. Corollary. If $G$ is an odd order finite group of rank equal to two, then it acts freely on a finite complex $X \simeq S^{n} \times S^{m}$.

\section{References}

[1] Adem, A., Davis, J. and Ünlü, Ö, Fixity and Free Group Actions on Products of Spheres, Comm. Math. Helv. 79, pp. 758-778 (2004).

[2] Adem, A. and Karagueuzian, D., Essential Cohomology of Finite Groups, Comm. Math. Helv. 72 (1997), pp. 101-109.

[3] A. Adem and R.J. Milgram, Cohomology of Finite Groups, Springer-Verlag Grundlehren 309(2004).

[4] A. Adem and R.J. Milgram, The Mod 2 Cohomology Rings of Rank 3 Simple Groups are Cohen-Macaulay, Annals of Math Studies 138, pp. 3-13, Princeton University Press (1995).

[5] Adem, A. and Pakianathan, J., On the Cohomology of Central Frattini Extensions, Journal of Pure and Applied Algebra 159, pp. 1-14 (2001)

[6] Adem, A. and Smith, J., Periodic Complexes and Group Actions, Annals of Mathematics 154 (2001), pp. 407-435.

[7] Alperin, J. and Evens, L., Representations, resolutions and Quillen's dimension theorem, J.Pure \& Applied Algebra 22 (1981), pp. 1-9.

[8] Artin, E. and Tate, J., Class Field Theory Benjamin Publ., 1968.

[9] Benson, D. Representations and Cohomology, vols. I, II. (second edition) Cambridge University Press, Cambridge (1998).

[10] Benson, D. and Carlson, J., Complexity and Multiple Complexes, Math. Zeitschrift 195 (1987), pp. 221-238.

[11] Benson D. and Carlson, J., Projective resolutions and Poincaré duality complexes, Trans. Amer. Math. Soc. 342 (1994), pp. 447-488.

[12] Browder, W., Cohomology and group actions, Inv.Math. 71 (1983), pp. 599-608.

[13] Brown, K. S., Cohomology of Groups Graduate Texts in Mathematics, 87 Springer-Verlag New York, (1982).

[14] Carlson, J., Depth and Transfer Maps in the Cohomology of Groups, Math. Zeit. 218 (1995), pp. 461-468.

[15] Carlson, J., Cohomology and induction from elementary abelian subgroups Quarterly J. Math. (Oxford) 51 (2000), pp. 169-181.

[16] Carlsson, G., On the Nonexistence of Free Actions of Elementary Abelian Groups on Products of Spheres, Amer. Journal Math. 102 (1980), pp. 1147-1157.

[17] Carlsson, G., On the Rank of Abelian Groups Acting Freely on $\left(\mathbb{S}^{n}\right)^{k}$, Inventiones Mathematicae 69 (1982), pp. 393-400.

[18] Carlsson, G., Free $(\mathbb{Z} / 2)^{3}$ Actions on Finite Complexes, Annals of Math. Studies 113 (1987), pp. 332-344.

[19] Carlsson, G., Free $(\mathbb{Z} / 2)^{k}$ Actions and a Problem in Commutative Algebra, Lecture Notes in Math 1217 (1985), pp. 79-83.

[20] Cartan H. and Eilenberg, S., Homological Algebra, Princeton University Press, Princeton NJ (1956)

[21] Duflot, J., Depth and Equivariant Cohomology, Comm. Math. Helv. 56 (1981) pp. 627-637.

[22] Feshbach, M., The Mod 2 Cohomology of Symmetric Groups and Rings of Invariants, Topology 41 (2002), pp. 57-84.

[23] Heller, A., A Note on Spaces with Operators, Ill. J. Math. 3 (1959), pp. 98-100. 
[24] Jackson, M., Qd(p)-free rank two finite groups act freely on a homotopy product of two spheres preprint 2005 (math.AT/0503746).

[25] Madsen, I., Thomas, C.B. and Wall, C.T.C., The Topological Spherical Space Form Problem II, Topology 15 (1978), pp. 375-382.

[26] Milnor, J., Groups Which Act on $\mathbb{S}^{n}$ without Fixed Points, Amer.J.Math. 79 (1957), pp. 623 630.

[27] Nakaoka, M., Homology of the infinite symmetric group, Ann. Math. 73 (1961), pp. 229-257.

[28] Quillen, D., The spectrum of an equivariant cohomology ring I \& II, Ann. Math. 94 (1971), pp. 549-602.

[29] Quillen, D., On the cohomology and K-theory of the general linear groups over a finite field, Ann. Math. 96 (1972), pp. 552-586.

[30] Quillen, D., The mod 2 cohomology rings of extra-special 2-groups and the spinor groups, Math. Ann. 194 (1971), pp. 197-212.

[31] Quillen, D. and Venkov, B. Cohomology of finite groups and elementary abelian subgroups, Topology 11 (1972), pp. 317-318.

[32] Serre, J.-P. Sur la Dimension Cohomologique des Groupes Profinis, Topology 3 (1965) pp. 413-420.

[33] Smith, P.A., Permutable Periodic Transformations, Proc.Nat.Acad.Sci. 30 (1944), pp. 105108.

[34] Steenrod, N., The Topology of Fibre Bundles, Princeton University Press 1951.

[35] Suzuki, M., Group Theory II, Springer-Verlag, New York (1986).

[36] Swan, R.G., Periodic Resolutions for Finite Groups, Annals of Mathematics 72 (1960), pp. 267-291.

[37] Ünlü, Ö., Doctoral Dissertation, UW-Madison, 2004.

[38] Venkov, B. Cohomology algebras for some classifying spaces, Dokl. Akad. Nauk. SSR 127 (1959), pp. 943-944.

[39] Webb, P., A local method in group cohomology, Comm. Math. Helv. 62 (1987), pp. 135-167.

[40] Wolf, J. Spaces of Constant Curvature (5th edition), Publish or Perish, 1984.

Department of Mathematics, University of British Columbia, Vancouver BC V6T

1Z2, CANADA

E-mail address: adem@math.ubc.ca 